\title{
Derechos Humanos y dificultades del lenguaje oral en niños y niñas que asisten a la fundación "Mi Comunidad Previene"
}

\section{Human Rights and difficulties of oral language in children and girls attending the "My Community Prevents foundation"}

\author{
María Elena Carreño Acebo \\ marielena@hotmail.com \\ Universidad Laica Eloy Alfaro de Manabí \\ Ecuador \\ http://orcid.org/0000-0002-4939-6902 \\ Kevin Juan Joza Carreño \\ Kjozac64@hotmail.com \\ Universidad Laica Eloy Alfaro de Manabí \\ Ecuador \\ https://orcid.org/0000-0002-1856-618X
}

Recibido: 10 de abril de 2019

Aprobado: 08 de mayo de 2019

\section{RESUMEN}

La protección integral que el Estado, la Sociedad y la Familia deben garantizar a todos los niños y niñas, que viven en el Ecuador, han de lograr el desarrollo integral pleno de sus derechos, en un marco de libertad, dignidad y equidad, tal como lo establece la constitución. Sin embargo, existen un sin número de discapacidades, entre estas del lenguaje poco atendidas. Con base a este precepto, el presente artículo se propuso evaluar los derechos y dificultades del lenguaje oral en los niños y niñas que asisten para hacer valer su derecho de atención a la Fundación "Mi Comunidad Previene". Para ello, se utilizó una metodologia con un enfoque de tipo mixto, que implico herramientas de tipo cualitativo y cuantitativo. La población la constituyeron 15 sujetos, entre niñas y niños en edad escolar. Entre los resultados se pudo constatar, que el mayor hallazgo se correspondió en un $61 \%$ los trastornos en la pronunciación, presentado entre sus conclusiones que existe poca fluidez del lenguaje oral en 
términos de normalidad durante la producción de los sonidos y calidad de voz requerida para el habla.

Descriptores: Derechos en niños y niñas; dificultades del lenguaje oral; dificultades comunicativas y problemas genéticos.

\section{ABSTRACT}

The comprehensive protection that the State, Society and the Family must guarantee to all children, living in Ecuador, must achieve the full comprehensive development of their rights, within a framework of freedom, dignity and equity, as established by the constitution. However, there are few disabilities, including underserved language. On the basis of this provision, this article was proposed to evaluate the rights and difficulties of oral language in children who attend to assert their right of care to the "My Community" Foundation. To this end, a methodology was used with a mixed-type approach, which involved qualitative and quantitative tools. The population consisted of 15 subjects, including school-age children. Among the results it could be noted, that the largest finding corresponded to $61 \%$ of the disturbances in pronunciation, presented among its conclusions that there is little fluency of oral language in terms of normality during the production of sounds and quality voice required for speech.

Descriptors: Rights in children, Oral language issues, Communication difficulties and genetic problems.

\section{INTRODUCCION}

Se estima que 150 millones de niños a nivel mundial poseen algún tipo de discapacidad y que el $80 \%$ de ellos se encuentra en países en vías de desarrollo. En la mayoría de los casos, estos niños no reciben el tratamiento que necesitan y muchos son discriminados.

En este sentido, la Convención sobre los Derechos de las Personas con Discapacidad, adoptada en el año 2006, y atendiendo a la discapacidad conceptualmente como: "Las personas con discapacidad incluyen a aquellas que tengan deficiencias físicas, mentales, intelectuales o sensoriales a largo plazo que, al interactuar con diversas barreras, éstas puedan impedir su participación plena y efectiva en la sociedad y en igualdad de condiciones con las demás." Es de 
indicar, que ya para el año 1989, en el primer tratado internacional incluye una referencia específica a la discapacidad y no discriminación de los niños con discapacidad y los cuales refuerzan , en su articulado 23, en la que exponen: "Los Estados Partes reconocen que el niño mental o físicamente impedido deberá disfrutar de una vida plena y decente en condiciones que aseguren su dignidad, le permitan llegar a valerse por sí mismo y faciliten la participación activa del menor en la comunidad."

Ante ello, la fundación "Mi Comunidad Previene", atiende este derecho en los niños que asisten a su organización, para darle asistencia temprana de las dificultades en niños de su comunidad. En este artículo, se presentan los resultados del trabajo realizado en 15 niños con problemas de lenguaje, en correspondencia al derecho a su salud consagrado en la constitución de la república del Ecuador, para ello se estableció como objetivo vincular los derechos y las dificultades del lenguaje oral en niños y niñas que asisten a la fundación mi comunidad previene

\section{DESARROLLO}

\section{Derechos del niño discapacitado}

A menudo, la discapacidad de un niño es seguida por su marginación en la sociedad. Por ejemplo, tan solo alrededor de un $2 \%$ de los niños con discapacidad tiene acceso a la educación. Además, la discapacidad impresiona a la gente. En la conciencia colectiva existe con frecuencia la creencia de que las personas con discapacidad sufren una maldición o castigo provocado por los pecados que sus ancestros cometieron en el pasado. En muchas regiones de todo el mundo, los niños que sufren una discapacidad son aislados, rechazados y se consideran intocables.

Sobre todo, se trata de un hecho evidente en los países en vías de desarrollo, donde la mayoría de los niños con discapacidad son analfabetos y viven totalmente aislados del resto de la sociedad. En muchos casos, estos menores se 
ven obligados a mendigar para poder sobrevivir y viven en la calle en situaciones de extrema pobreza.

\section{Derechos de los niños con discapacidad}

Los niños con discapacidad son iguales al resto de niños. De acuerdo con la Convención sobre los Derechos del Niño (CDN, 2013), los menores con discapacidad poseen los mismos derechos que cualquier otro niño. Además del derecho a la no discriminación previamente mencionado, es necesario destacar que los niños con discapacidad deben disfrutar de los siguientes derechos:

\section{Derecho a un tratamiento adecuado}

El segundo párrafo del Artículo 23 de la CDN, dedicado a los niños con discapacidad, garantiza su derecho a obtener una asistencia especial y a solicitar la concesión de una subvención del gobierno que se ajuste en función del país del niño y de la situación financiera de sus padres o tutor.

\section{Derecho a la educación}

Debido a la falta de infraestructuras, medios, conocimiento y, sobre todo, de buena voluntad, la mayoría de los niños con discapacidad no recibe ninguna educación; en realidad, ni siquiera una educación primaria. De hecho, de acuerdo con una serie de estadísticas internacionales, solo el $2 \%$ de los niños con discapacidad tiene el privilegio de asistir a la escuela.

\section{Derecho al ocio}

En ocasiones, las personas que rodean a los niños con discapacidad están tan involucradas en su asistencia que olvidan que éstos, antes que nada, son niños; niños que necesitan divertirse, jugar, expresarse de forma artística, practicar deportes, etc. 


\section{Derecho a la opinión}

Se trata de una de las necesidades fundamentales de un niño con discapacidad, aunque desafortunadamente, también es una de las más ignoradas. El derecho a la opinión se extiende del mismo modo a todos los ámbitos de la vida social, en la que el niño debería contar con la oportunidad de expresar su opinión, ser consultado e informado y tener la oportunidad de participar.

Todos los derechos mencionados, se encuentran en la mayoría de los casos cercenados en los derechos de los niños con discapacidad del lenguaje oral.

\section{Tipos de dificultades del lenguaje oral}

Alteraciones del lenguaje: Aguilera (2017), lo explica como un sistema complejo, estructurado y simbólico que comprende diferentes subsistemas, debido a la falta del habla, habla ininteligible y errores para construir oraciones asimismo el lenguaje alterado puede afectar a la expresión solamente, o también a la comprensión de las sustituciones de palabras difíciles por otras más sencillas; no pronunciar la parte final de ciertas palabras; palabras incompletas; omisión o cambio de vocales.

Trastornos de la comprensión: Navarro (2009), indica que es un trastorno grave y duradero que afecta a la adquisición del lenguaje desde sus inicios, se prolonga durante la infancia y la adolescencia, pudiendo dejar en algunos casos secuelas significativas en la edad adulta.

Trastornos de la pronunciación: Este trastorno se caracteriza principalmente en que el niño omite o pronuncia los fonemas de forma inadecuada (distorsiones, sustituciones) para su edad mental aunque tenga un nivel normal para el resto de las funciones del lenguaje. 
Trastornos del habla o expresión verbal: Los trastornos del habla pueden constituir problemas con la formación de sonidos, los cuales se llaman trastornos de la articulación o fonológicos, o pueden incluir dificultades con la intensidad con el tono, volumen, o calidad de la voz. (Torres, 2001).

Estos trastorno específicos del lenguaje en niños y niñas, basándose en sus aspectos fonológicos, morfológicos, sintácticos, semánticos y pragmáticos. Pueden tener las características de comportamiento y lingüísticas muy parecidas al autismo, pero lo diferenciaremos por su capacidad de socializar. (Juárez y col, 2013).

\section{Causas de las dificultades en el desarrollo del lenguaje}

Para Campo (2009), se pueden distinguir las siguientes:

Hipoacusia o sordera: Ésta se puede detectar desde muy pequeños ya que el niño con hipoacusia severa prácticamente no balbuceará, si ésta no es severa o profunda se puede detectar en la segunda o tercera fase de desarrollo del lenguaje ya que producirá ruidos como gritos sin sentido alguno cada vez que quiera expresar algo. Si se detecta alguno de estos síntomas es importante acudir lo antes posible con un especialista en audiología para que se le realice una valoración completa y se proceda con los métodos de rehabilitación.

Alteraciones en la discriminación auditiva: Se presenta cuando existen fallas o alteraciones en el nervio auditivo o en la corteza cerebral que impiden la discriminación de fonemas, el niño empieza a hablar mal, a sustituir sonidos o tiende a mantenerse callado. Si esto sucediera es importante llevar al pequeño a atención especializada con una terapeuta de lenguaje lo antes posible.

Fallas en la comprensión: Los problemas de lenguaje se pueden deber a fallas en la comprensión, ya que el lenguaje implica: Escuchar un sonido; evocar una 
imagen mental; transformar la imagen mental; producir un sonido. Cuando existen fallas de abstracción el niño no puede realizar este proceso, por lo que se limitará a pronunciar o a decir lo que puede tocar en ese momento. Es común en estos niños que las órdenes más simples se les olviden, no recuerden cosas sencillas como el nombre de sus padres y no obedezcan continuamente. Este problema puede ser rehabilitado por un especialista en Comunicación Humana o Terapeuta de Lenguaje, sin embargo, requiere de atención durante un periodo largo.

Fallas en la articulación: Este problema se da cuando el pequeño no puede pronunciar fonemas que deberían de estar ya superados para su edad. Lo que generalmente sucede es que el pequeño acomoda los músculos de la lengua y las mejillas de manera incorrecta y por lo tanto el sonido que produce no coincide con el que quisiera producir. Por lo general estos niños se dan cuenta de que hablan mal y para no pasar malos ratos tratan de no hablar.

Tartamudez: Considerado una causa emocional, se presenta como un problema de habla, no de lenguaje propiamente dicho, conviene advertir que existe un periodo evolutivo de tartamudez, no todos los niños lo pasan pero sí un gran porcentaje, éste comprende entre los tres y cinco años. Se recomienda no presionarlos, nunca completarles las frases y ayudarlos a que se relajen y respiren para que puedan hablar pausadamente. Si esta fase se extiende se recomienda acudir con un especialista.

Causa física o funcional: Existe otro grupo de problemas de lenguaje que se encuentran determinados por alteraciones físicas o funcionales del aparato fonoarticulador, en este caso el más indicado para su detección y solución es el pediatra. Es importante que la rehabilitación se lleve a cabo inmediatamente después de que el problema ha sido detectado, ya que entre más pequeño sea el niño más rápido resolverá su problemática, así mismo, se pueden evitar los 
problemas que estas situaciones traen consigo, tales como alteraciones emocionales, inseguridad y hasta mutismo (silencio absoluto). Otra ventaja de corregir a tiempo los problemas de lenguaje es que se evitan complicaciones futuras en el momento de la adquisición de la lecto-escritura.

Estimulación insuficiente: El lenguaje se adquiere por imitación y requiere de una incentivación importante por parte de los padres, por lo que no es de extrañar que un niño al cual se le habla lo estrictamente indispensable y no se le invita constantemente a que él también lo haga o no se le presta atención cuando intenta hacerlo y sólo logra ser atendido cuando llora o grita, pierda todo interés en comunicarse verbalmente.

\section{METODOLOGIA}

La investigación utilizó un método mixto, que implico un enfoque tanto cualitativo como cuantitativo. (Sampieri, 2014). El enfoque cualitativo, se basó en métodos de recolección de datos, sin medición numérica, como las descripciones y observaciones dadas por los padres de los niños y niñas investigados, lo que permitió determinar el estudio de los derechos y las dificultades del lenguaje oral en niños y niñas que asisten a la fundación "Mi Comunidad Previene". Por otro lado, el enfoque cuantitativo se utilizó en la recolección y el análisis de los datos aportados por las evaluaciones realizadas y de esta manera conocer con exactitud patrones de comportamiento de la población. La muestra estuvo constituida por 15 sujetos, entre niñas y niños en edad escolar. Teniendo a consideración estas características, ambos enfoques resultaron muy valiosos para esta investigación, puesto que combino técnicas para la recolección de información, además de, constituir diferentes aproximaciones al estudio de los problemas del lenguaje. 


\section{RESULTADOS}

Posterior al análisis de los resultados obtenidos a la evaluación en 15 niños en niños y niñas que asisten a la fundación "Mi Comunidad Previene", se presentan las siguientes tablas y análisis:

Tabla 1. Dificultades comunicativas que presentan los niños y niñas que asisten a la fundación mi comunidad previene.

\begin{tabular}{|l|l|l|}
\hline Tipo Comunicativas & Fr & $\%$ \\
\hline Tartamudeo & 2 & 13 \\
\hline Tono de voz muy bajo & 7 & 47 \\
\hline $\begin{array}{l}\text { Mal formaciones labiales o } \\
\text { mandibulares }\end{array}$ & 2 & 13 \\
\hline Se aísla socialmente & 4 & 27 \\
\hline Total & 15 & 100 \\
\hline
\end{tabular}

Fuente: elaboración propia.

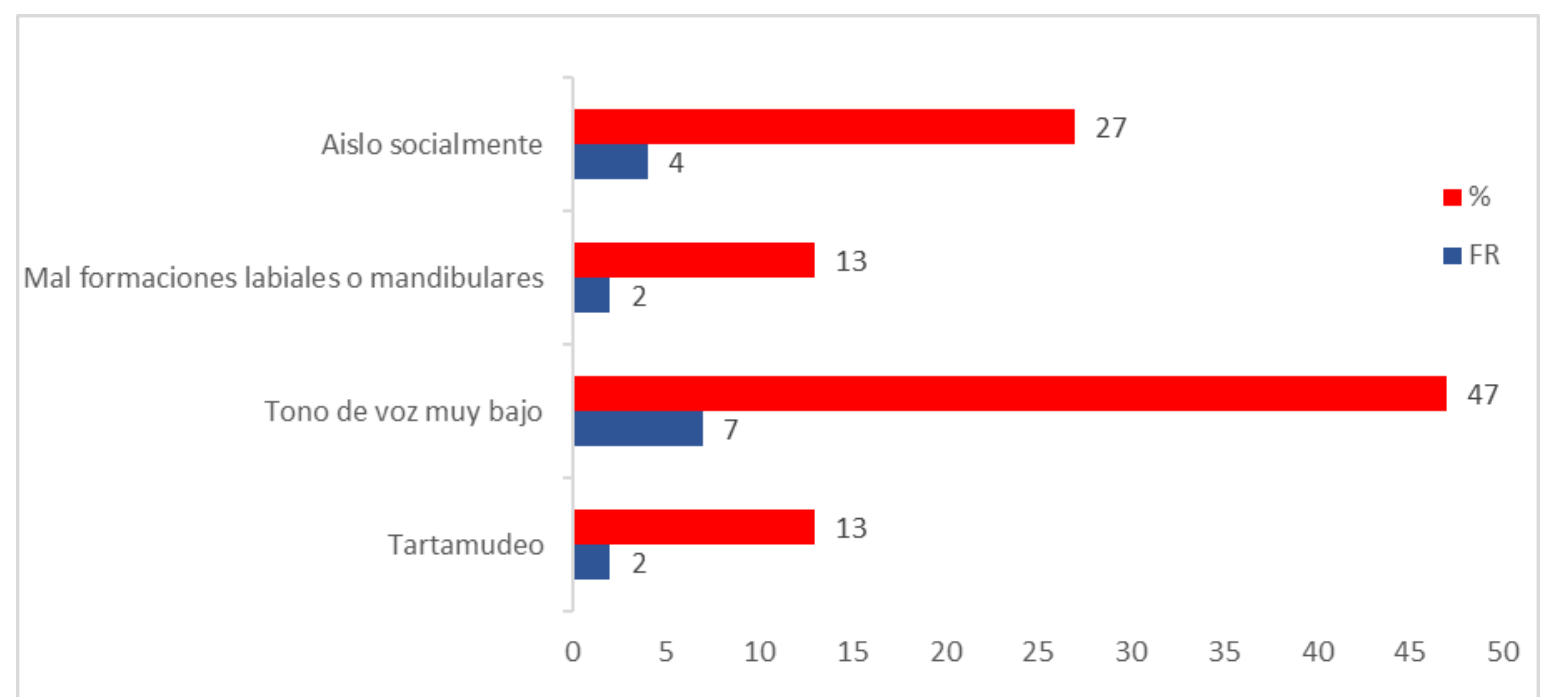

Grafico 1. Dificultades comunicativas que presentan los niños y niñas que asisten a la fundación "Mi Comunidad Previene".

Fuente: elaboración propia. 
En la tabla y grafico 1, se presentan los resultados del análisis de las dificultades comunicativas que presentan los niños y niñas que asisten a la fundación "Mi Comunidad Previene", en la que se indica que el $47 \%$ se evaluaron por el tono de voz muy bajo, seguido del $27 \%$ que manifestaron aislamiento social y con el $13 \%$ por igual para las dificultades de tartamudeo y mal formaciones labiales o mandibulares. Es de indicar, que estas dificultades están directamente relacionadas con la producción de los sonidos y calidad de voz requerido para el habla. Asimismo, el test de evaluación aplicado determinó que los niños tartamudean al momento de expresarse, consecuente a que no tienen una buena fluidez y tienden a sentir vergüenza al expresarse.

Tabla 2. Tipos de dificultades de lenguaje oral que poseen los niños y niñas que asisten a la fundación "Mi Comunidad Previene"

\begin{tabular}{|l|l|l|}
\hline Tipos /Análisis & Fr & \% \\
\hline Alteraciones del lenguaje & 3 & 19 \\
\hline $\begin{array}{l}\text { Trastornos de la } \\
\text { pronunciación }\end{array}$ & 8 & 53 \\
\hline Trastornos del habla & 4 & 28 \\
\hline Total & 15 & 100 \\
\hline
\end{tabular}

Fuente: Elaboración propia 


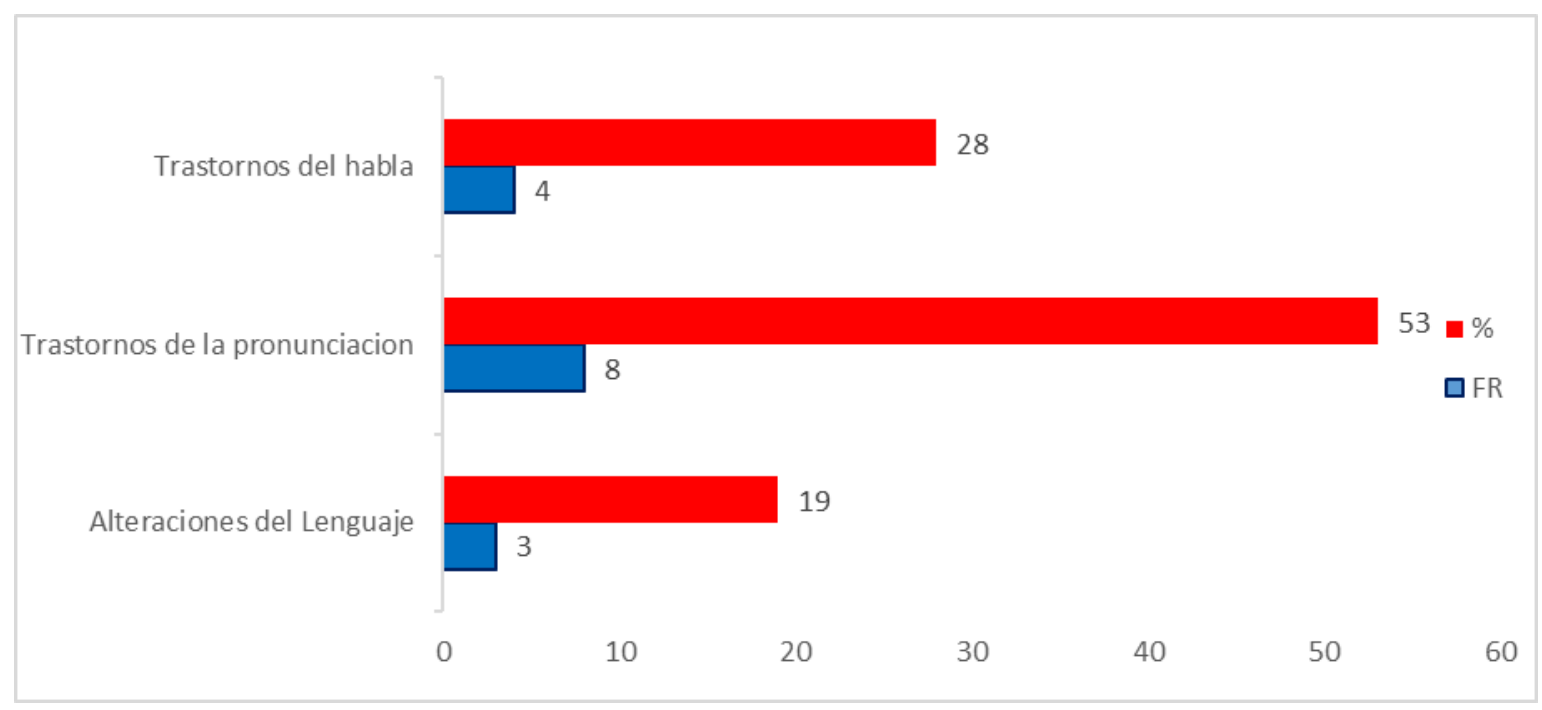

Grafico 2. Tipos de dificultades de lenguaje oral que poseen los niños y niñas que asisten a la fundación "Mi Comunidad Previene".

Fuente: Elaboración propia.

En la tabla y grafico 2, se presentan los resultados del análisis de los tipos de dificultades de lenguaje oral que poseen los niños y niñas que asisten a la fundación "Mi Comunidad Previene", en la que se indica que el $61 \%$ se evaluaron con trastornos en la pronunciación, seguido del $22 \%$ que se les diagnostico trastornos en el habla y el menor porcentaje con el $13 \%$ presento alteraciones en el lenguaje. Los resultados expuestos, permite indicar a los trastornos de pronunciación con mayor incidencia en los niños y niñas en edad escolar, identificados por su poca fluidez del lenguaje oral en términos de normalidad. Cabe destacar, que estas dificultades están directamente relacionadas con la producción de los sonidos y calidad de voz requerida para el habla. 
Tabla 3. Tipos de problemas genéticos de lenguaje oral que poseen los niños y niñas que asisten a la fundación "Mi Comunidad Previene".

\begin{tabular}{|l|l|l|}
\hline Tipos /Análisis & Fr & \% \\
\hline Frenillo & 7 & 47 \\
\hline Labio leporino & 0 & 0 \\
\hline Deficiencia Auditiva & 5 & 34 \\
\hline Ninguna de las anteriores & 3 & 19 \\
\hline
\end{tabular}

Fuente: Elaboración propia

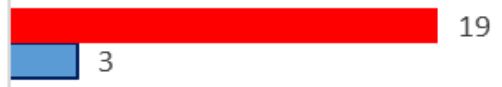

Deficiencia Auditiva

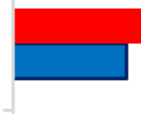

5

Labio leporino

Frenillo

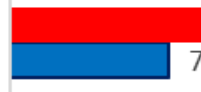

7

0

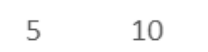

15

20

Grafico 3. Tipos de problemas genéticos de lenguaje oral que poseen los niños y niñas que asisten a la fundación "Mi Comunidad Previene".

Fuente: Elaboración propia

En la tabla y grafico 3 , se presentan los resultados del tipo de problemas genéticos del lenguaje oral que poseen los niños y niñas que asisten a la fundación "Mi Comunidad Previene", en la que se indica que el $47 \%$ se diagnosticaron con frenillo como impedimento para su pronunciación, seguido del $34 \%$ que se les diagnostico deficiencia auditiva y con el $19 \%$ no presentó ninguna 
de las alteraciones previstas en la evaluación. Dichos problemas genéticos, son factores que impiden tener un correcto desarrollo del lenguaje oral, debido a las alteraciones que generan en los órganos que intervienen en la protección del lenguaje, así como en su fluidez y normal desempeño en los niños y niñas evaluados. Es de indicar que no se presentaron casos de dificultad del lenguaje por diagnóstico de labio leporino. Este hecho, fue corroborado por los representantes de los niños y niñas en edad escolar, convirtiéndose en la principal razón de su correcta pronunciación causando un retraso en su vida social y en el nivel de aprendizaje escolar.

\section{CONCLUSIONES}

Posterior al análisis y la presentación de los resultados de la investigación, relacionados con los derechos y las dificultades del lenguaje oral en niños y niñas que asisten a la fundación "Mi Comunidad Previene", se pudo concluir:

Existe un cumplimiento del derecho de los niños y niñas por intermedio de las acciones sociales emprendidas por la Fundación "Mi Comunidad Previene", quienes brindaron atención prioritaria y pertinente para aquellos evaluados con discapacidad oral, a través de los ejercicios de pronunciación, expresión y los de articulación.

Se evidencio un predominio de tono de voz muy bajo, entre los niños y niñas evaluados, advertidos a través de los problemas de producción de los sonidos y la voz durante el habla, lo que les impide una buena fluidez, generándoles trastornos de pronunciación con mayor incidencia en los niños y niñas en edad escolar durante el lenguaje oral en términos de normalidad.

Entre los tipos de dificultades del lenguaje oral, se constató con mayor dominio los trastornos en la pronunciación, los cuales están identificados por la poca fluidez del lenguaje oral en términos de normalidad durante la producción de los sonidos y calidad de voz requerida para el habla. 
Dentro de los problemas congénitos de lenguaje oral que privan en los niños y niñas evaluados, se diagnosticó el frenillo en la mayoría de los casos, como impedimento para su pronunciación. Sin embargo la deficiencia auditiva se posesiono con un nivel representativo, ambos problemas impiden el correcto desarrollo del lenguaje oral por las alteraciones de tipo orgánico que intervienen en la protección del lenguaje, así como en su fluidez y normal desempeño en los niños y niñas evaluados.

Estos aspectos sin duda alguna permiten un mejor reconocimiento de los Derechos de los Niños y Niñas de forma integral dentro de su ambiente natural; dignificando su presencia en la misma. Al respecto de lo anterior señalan Aldana e Isea (2018):

Los derechos humanos y la dignidad contemplan una relación intrínseca, articulándose una correlación entre ambos, en la medida que los derechos humanos son operacionalizados por las personas, en esa medida la dignidad humana se enaltece, mientras que al existir dignidad humana, se hacen presentes los derechos humanos.

Es así, que dentro del contexto desde donde se ha desarrollado la investigación, se debe siempre apuntalar hacia la dignificación de la persona, de los niños y niñas; para el reconocimiento de sus derechos dentro de la sociedad.

\section{REFERENCIAS CONSULTADAS}

1. Aguilera Albesa S. y Orellana Ayala C.E. (2017). "Trastornos del lenguaje". Pediatría Integral. XXI.

2. Aldana Zavala, J., \& Isea, J. (2018). Derechos Humanos y Dignidad Humana. IUSTITIA SOCIALIS, 3(4), 8-23. Recuperado de http://fundacionkoinonia.com.ve/ojs/index.php/lustitia Socialis/article/view/1 $\underline{19 / 101}$

3. Campo, L (2009).Características del desarrollo cognitivo y del lenguaje en niños de edad preescolar. Psicogente, 12(2), 341-351. 
4. CDN. (2013). Convención sobre los Derechos del Niño. Recuperado en: www.un.org/es/events/childrenday/pdf/derechos.pdf

5. Juárez, J y Bonilla, M (2013).Corrección neuropsicológica en las dificultades de la expresión y la comprensión del lenguaje. Pensamiento Psicológico, $12(1), 113-127$.

6. Navarro, M. (2009). Adquisición del lenguaje. El principio de la comunicación. Revista de Filología y su Didáctica, 26 (1), 321-347.

7. Sampieri Hernandez Roberto. (2014). "Metodología de la investigación". (en línea). Consultado 14 de Agosto 2017. Recuperado en: http://metodoscomunicacion.sociales.uba.ar/files/2014/04/Hernandez-Sampieri-Cap-1.pdf

8. Torres, Z (2001) Trastornos del habla o expresión verbal. Barcelona: Siegel.

\section{REFERENCES CONSULTED}

1. Aguilera Albesa S. and Orellana Ayala C.E. (2017). "Language disorders." Integral Pediatrics XXI.

2. Aldana Zavala, J., \& Isea, J. (2018). Human Rights and Human Dignity. IUSTITIA SOCIALIS, 3 (4), 8-23. Retrieved from http://fundacionkoinonia.com.ve/ojs/index.php/lustitia_Socialis/article/view/1 $19 / 101$

3. Campo, $L$ (2009). Characteristics of cognitive and language development in preschoolers. Psychogent, 12 (2), 341-351.

4. CRC (2013) Convention on the Rights of the Child. Retrieved from: www.un.org/es/events/childrenday/pdf/derechos.pdf

5. Juárez, J and Bonilla, M (2013). Neuropsychological correction in the difficulties of expression and understanding of language. Psychological Thought, 12 (1), 113-127.

6. Navarro, M. (2009). Acquisition of language. The principle of communication. Journal of Philology and its Didactics, 26 (1), 321-347. 
7. Sampieri Hernández Roberto. (2014) "Research methodology." (online). Accessed August 14, 2017. Retrieved from: http://metodoscomunicacion.sociales.uba.ar/files/2014/04/Hernandez-Sampieri-Cap-1.pdf

8. Torres, Z (2001) Speech disorders or verbal expression. Barcelona: Siegel.

(C2019 por el autor. Este artículo es de acceso abierto y distribuido según los términos y condiciones de la licencia Creative Commons Atribución-NoComercial-Compartirlgual 4.0 Internacional (CC BY-NC-SA 4.0) (https://creativecommons.org/licenses/by-nc-sa/4.0/). 